Portland State University

PDXScholar

Electrical and Computer Engineering Faculty

Publications and Presentations

Electrical and Computer Engineering

$1-1-1999$

Grazing Reflection of Gaussian Beams

Lee W. Casperson

Portland State University

Follow this and additional works at: https://pdxscholar.library.pdx.edu/ece_fac

Part of the Electrical and Computer Engineering Commons

Let us know how access to this document benefits you.

Citation Details

Lee W. Casperson, "Grazing Reflection of Gaussian Beams," Appl. Opt. 38, 554-562 (1999).

This Article is brought to you for free and open access. It has been accepted for inclusion in Electrical and Computer Engineering Faculty Publications and Presentations by an authorized administrator of PDXScholar. Please contact us if we can make this document more accessible: pdxscholar@pdx.edu. 


\title{
Grazing reflection of Gaussian beams
}

\author{
Lee W. Casperson
}

\begin{abstract}
The reflectivities of most surfaces are higher for grazing or near-90-deg angles of incidence than for more perpendicular or near-zero-deg angles. Grazing-incidence configurations are especially important in the development of lasers and optical systems that operate in the far-ultraviolet and soft-x-ray regions of the spectrum, where transparent or highly reflecting media are almost unknown. Analytical solutions of the paraxial wave equation are obtained for the grazing reflection and complex interference effects that take place when a Gaussian beam interacts at shallow angles with a reflecting surface. (C) 1999 Optical Society of America
\end{abstract}

OCIS codes: $140.0140,240.0240,260.0260,350.5500$.

\section{Introduction}

It has long been recognized that Gaussian beams, and more generally polynomial-Gaussian beams, are solutions of the paraxial wave equation in free space. Such modes also arise frequently as the output beams in laser oscillators. Accordingly, there has been intense and continuing interest in the propagation and transformation of Gaussian beams of various wavelengths in refracting, reflecting, and diffracting systems. Over the years many such systems have been investigated. It was shown that Gaussian beams retain their basic functional form when they propagate in free space, ${ }^{1}$ in real lenslike media, ${ }^{2}$ in complex lenslike media, ${ }^{3}$ and in other simple elements. ${ }^{4}$ Offaxis polynomial-Gaussian beams have also been studied, ${ }^{5}$ and their propagation in a range of complex and misaligned systems is now known. ${ }^{6}$

One of the oldest problems in electromagnetics concerns the reflection of electromagnetic waves at boundaries, and this problem has been studied extensively in the Gaussian beam context. The grazing reflection of Gaussian beams is investigated here. At grazing incidence the beam might interact strongly with a reflecting surface for a distance comparable with or longer than the Rayleigh length.

When this research was performed the author was with the Rochester Theory Center for Optical Science and Engineering and the Institute of Optics, University of Rochester, Rochester, New York 14627-0186. His permanent address is the Department of Electrical Engineering, Portland State University, P.O. Box 751, Portland, Oregon 97207-0751. His email address is lcaspers@ ee.pdx.edu.

Received 6 July 1998.

0003-6935/99/030554-09\$15.00/0

(C) 1999 Optical Society of America
Thus the meaning and usefulness of conventional beam parameter transformations are not so clear. The actual electromagnetic-field distributions of the reflecting waves are obtained here, including the interference terms between the incident and the reflected waves. For near-normal incidence the intensity or energy density maxima that result from this interference are separated by approximately one half of the wavelength, a distance that might be small compared with most of the macroscopic components of an electromagnetic system. The existence and dimensions of these fringes would be of interest in applications that involve nonlinear media, and they have made possible the enhanced resolution of interference scanning optical microscopy. ${ }^{7}$ In most previous studies of non-normal-incidence reflection the interference between the incident and the reflected waves is not treated, and only the beam parameter transformations are obtained. However, it can be noted that, as the angle of incidence increases, the spacing between the interference fringes also increases. At grazing incidence the interference fringes that result from the superposition of the incident and the reflected waves can be comparable in width to the Gaussian beam spot size (1/e amplitude radius). In spite of this apparent complication, it is shown here that solutions of the paraxial equation can be found for grazing reflection of a Gaussian beam.

An analysis of the grazing reflection of Gaussian beams can have significant practical implications. One of the most important reasons for interest in grazing reflectivity is the often dramatic increase in the reflection coefficient that occurs as angles of incidence approach $90 \mathrm{deg}$, and this effect was studied long before the advent of lasers. ${ }^{8}$ This angle depen- 
dence might already be important for microwave and visible wavelengths, but it is most striking for wavelengths in the vacuum-ultraviolet spectral region. Although there might be other important advantages to employing grazing reflection, the higher reflectivities that are obtainable have been the motivation for many applications.

One of the earliest applications of grazing-incidence reflection was in vacuum-ultraviolet spectroscopy. The low reflectance of all grating materials for wavelengths below $\sim 300 \AA$ requires the use of grazingincidence spectrographs for this spectral region. In these systems the grating itself can be oriented to have an angle of incidence of $89 \mathrm{deg}$ or more. By this means useful reflectivities and grating efficiencies have been obtained at wavelengths as low as a few angstroms. To disperse still shorter wavelengths, as required in spectroscopy, the gratings have been replaced with crystals in which the regular lattice spacing takes the place of the rulings on a diffraction grating. Some spectroscopic systems have incorporated two or more grazing-incidence elements. ${ }^{9}$

With the development of more recent electromagnetic systems, several further applications of grazingincidence reflection have appeared. One important example is in the guiding of optical beams when no suitable fiber-optic material is available. For example, in the infrared and the far-infrared portions of the spectrum there are wavelength regions for which low-loss fiber materials are not yet readily available. Similarly, in the far-ultraviolet and the soft-x-ray regions, transparent materials are virtually unknown, whereas grazing reflectivities from metal can be quite high. For such wavelengths and for extremely highpower applications, metallic waveguides are sometimes a viable option. Thus, in the limit of a 90-deg angle of incidence, low-loss waveguided propagation is possible if there is a slight inward curvature of the waveguiding surface in the direction of propagation. ${ }^{10}$ If such a waveguiding strip is also curved appropriately in the transverse direction, it can support low-loss propagation of beam modes at wavelengths for which other media would be essentially opaque. ${ }^{11}$ The modes can sometimes be represented in terms of Hermite-Gaussian functions transverse to the waveguide and Airy functions in the perpendicular direction. If mirrors are placed on the ends of such a waveguide while an amplifying medium is introduced, it becomes possible to achieve laser oscillation. Such lasing has been observed with a continuous-wave $\mathrm{CO}_{2}$ laser in which the discharge tube was slightly bent ${ }^{12}$ and in a rf discharge for which the waveguide also served as one of the discharge electrodes. ${ }^{13}$ It has also been possible to match the mode volume of the fundamental Airyfunction mode to the thin gain layer along the curved cathode of a continuous-wave transverse laser discharge. ${ }^{14}$ This sort of coupling should also be possible for a laser with grazing reflection modes of the type described below.

Grazing reflection has also proved to be extremely useful in the development of high-power free-electron lasers. ${ }^{15-17}$ These lasers present difficulties because of the high power density of the optical beam that leaves the gain medium. Even though visible wavelength reflectivities can be quite high for normal incidence, it has been found that damage can be reduced substantially by a grazing-incidence orientation of the mirrors closest to the amplifier. ${ }^{18,19}$ Grazing-incidence components will be even more important with the development of free-electron lasers in the vacuum-ultraviolet and soft-x-ray regions of the spectrum. It is notable in the present context that Gaussian field distributions are often assumed when the modes of these grazing-incidence lasers are represented. ${ }^{20,21}$

Grazing-incidence mirrors are also used with other types of laser, and their potential as multibounce turning mirrors and polarizers for soft-x-ray lasers and optical systems has been recognized. ${ }^{22}$ Also, they can serve as an efficient means for collimating the soft-x-ray emission from a laser-produced plasma for longitudinal pumping of a short-wavelength laser. ${ }^{23}$ Thus grazing-incidence reflectors are finding their way into many applications, especially for systems that involve short wavelengths or high powers. In many of these applications the beam profiles can be well represented by Gaussian functions, and thus there is value in developing as complete a model as possible for the reflection of such beams at grazing angles of incidence.

A basic derivation of the Gaussian beam equations is included in Section 2. The initial purpose of this derivation is to reduce the partial differential wave equation to a set of first-order ordinary differential equations that govern the various parameters that characterize the spatially evolving beam. The solutions to these simpler equations are discussed in Section 3 for beam propagation in spatially homogeneous media. The grazing reflection of these beams is explored in Section 4.

\section{Derivation of the Beam Equations}

The geometry to be considered here involves a Gaussian beam that propagates almost parallel to a reflecting surface, as shown schematically in Fig. 1 . The coordinate system to be employed is also shown in Fig. 1. Owing to diffraction and misalignment, the beam is assumed to interact with the surface over some extended distance. The complexity of including this reflecting surface in the propagation analysis leads us to simplify the problem as much as possible in other ways. Thus the reflecting surface is assumed to be highly reflecting and flat, and other simplifications concern the propagation medium. With such restrictions it is possible to obtain closed-form analytic solutions that are not excessively cumbersome.

For any study of electromagnetic-wave propagation the fundamental starting point is the MaxwellHeaviside equations. These equations can be combined to yield coupled equations that govern the various field components of a propagating electromagnetic beam. For the case of nearly plane waves 


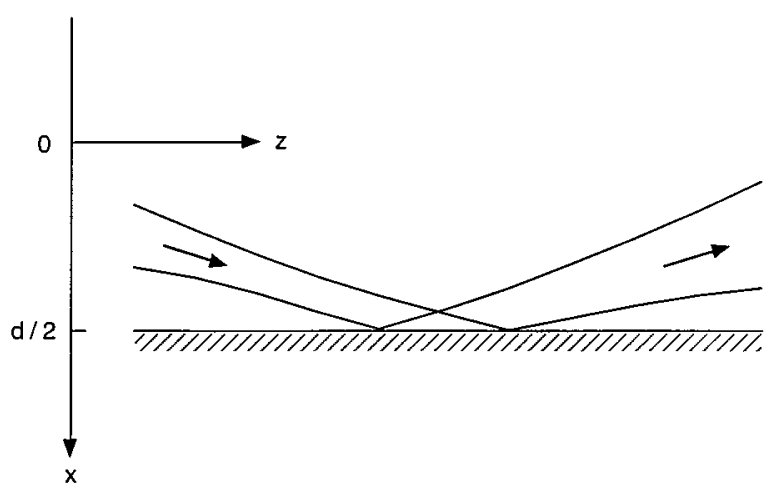

Fig. 1. Schematic representation of a Gaussian beam undergoing grazing reflection from a flat surface. The coordinate system used in the analysis is also shown.

in a medium in which the changes in permittivity and permeability per wavelength are small, the dominant transverse Cartesian field components are governed by the much simpler wave equation

$$
\nabla^{2} \mathbf{E}^{\prime}(x, y, z)+k^{2}(x, y, z) \mathbf{E}^{\prime}(x, y, z)=0,
$$

where $\mathbf{E}^{\prime}$ is the complex amplitude of the electric field and $k$ is the potentially complex spatially dependent wave number. The wave number could, in principle, have an imaginary part, owing to nonzero conductivity or out-of-phase components of the polarization or magnetization. Although Eq. (1) governs the dominant transverse components, the weak $z$ components of the fields can be found at any time from the transverse components by means of the MaxwellHeaviside equations.

In many practical situations, $k^{2}$ has at most quadratic spatial variations in the vicinity of the beam. However, no particular insight is gained by retention of propagation effects in both of the transverse directions, and thus we write at the outset

$$
k^{2}(x, y, z)=k_{0}(z)\left[k_{0}(z)-k_{1 x}(z) x-k_{2 x}(z) x^{2}\right] .
$$

Although the detailed solutions are for a spatially homogeneous medium $k(x, y, z)=k_{0}$, this more general starting point provides a guide for the study of other configurations. The wave number is assumed to be a complex function that is separable into its real and imaginary parts in the form $k=\beta+i \alpha$. For a linearly polarized wave that propagates primarily in the $z$ direction a useful substitution is

$$
E^{\prime}(x, y, z)=A(x, y, z) \exp \left[-i \int k_{0}(z) \mathrm{d} z\right],
$$

where $A$ is a new complex amplitude function. With this substitution Eq. (1) reduces to the paraxial wave equation

$$
\frac{\partial^{2} A}{\partial x^{2}}+\frac{\partial^{2} A}{\partial y^{2}}-2 i k_{0} \frac{\partial A}{\partial z}-i \frac{\mathrm{d} k_{0}}{\mathrm{~d} z} A-k_{0}\left(k_{1 x} x+k_{2 x} x^{2}\right) A=0,
$$

where $A$ is assumed to vary so slowly with $z$ that its second derivative can be neglected.

A useful form for a fundamental astigmatic off-axis Gaussian beam is ${ }^{24}$

$$
A(x, y, z)=A_{0} \exp \left\{-i\left[\frac{Q_{x}(z) x^{2}}{2}+S_{x}(z) x+P(z)\right]\right\},
$$

where for simplicity it is also assumed that the beam is uniform in the $y$ direction. The size of the beam and the curvature of the phase fronts are governed by the complex beam parameter $Q_{x}$. The location of the beam depends on the complex displacement parameter $S_{x}$, and the phase and amplitude of the beam are governed by the complex phase parameter $P$. If Eq. (5) is substituted into Eq. (4), by equating equal powers of $x$ one finds that the various parameters of the beam are governed by the following equations:

$$
\begin{aligned}
Q_{x}^{2}+k_{0} \frac{\mathrm{d} Q_{x}}{\mathrm{~d} z}+k_{0} k_{2 x} & =0, \\
Q_{x} S_{x}+k_{0} \frac{\mathrm{d} S_{x}}{\mathrm{~d} z}+\frac{k_{0} k_{1 x}}{2} & =0, \\
\frac{\mathrm{d} P}{\mathrm{~d} z} & =-i \frac{Q_{x}}{2 k_{0}}-\frac{S_{x}^{2}}{2 k_{0}}-\frac{i}{2 k_{0}} \frac{\mathrm{d} k_{0}}{\mathrm{~d} z} .
\end{aligned}
$$

The detailed significance of the parameter $Q_{x}$ is contained in the relation

$$
Q_{x}(z)=\frac{\beta_{0}(z)}{R_{x}(z)}-i \frac{2}{w_{x}^{2}(z)},
$$

where $\beta_{0}$ is the real part of the on-axis propagation constant and $R_{x}$ and $w_{x}$ are the radius of curvature of the phase fronts and the 1/e amplitude spot size in the $x$ direction, respectively. From Eq. (5) the ratio $d_{x a}=-S_{x i} / Q_{x i}$ is the displacement in the $x$ direction of the amplitude center of the Gaussian part of the beam, and the ratio $d_{x p}=-S_{x r} / Q_{x r}$ is the displacement in the $x$ direction of the phase center of the beam. The subscripts $i$ and $r$ denote the imaginary and real parts, respectively, of the parameters $Q_{x}$ and $S_{x}$.

It is convenient to express the complex displacement parameter in terms of the more physically intuitive amplitude displacement and the rate of change of this displacement with the propagation distance $z$. Combining Eqs. (6) and (7) with the displacement definitions, one obtains the relation ${ }^{25}$

$$
\begin{aligned}
S_{x}(z)= & -Q_{x}(z) d_{x a}(z)+\beta_{0}(z)\left\{d_{x a}^{\prime}(z)-\left[\alpha_{2 x}(z) d_{x a}(z)\right.\right. \\
& \left.\left.+\alpha_{1 x}(z) / 2\right] / Q_{x i}(z)\right\},
\end{aligned}
$$

where $\alpha_{2 x}$ is the imaginary part of $k_{2 x}, \alpha_{1 x}$ is the imaginary part of $k_{1 x}, d_{x a}^{\prime}$ represents the slope of the beam axis with respect to the $z$ axis, and it has been assumed that the gain per wavelength is small $\left(\alpha_{0} \ll\right.$ $\left.\beta_{0}\right)$. A similar equation would hold for $S_{y}(z)$ in a more general configuration. 
Many solutions have been obtained for the parameters governed by Eqs. (6)-(8). The evolution of the beam parameter $Q_{x}$ (or accordingly the spot size and phase front curvature) has been examined in the greatest depth, whereas much less attention has been given to the detailed solutions of the displacement parameter equations and their applications. No detailed studies of the phase parameter for general offaxis Gaussian beams appear to have been made. Because interference effects are essential for the study of the intensity distribution of a reflecting Gaussian beam, it is necessary here also to obtain rigorous solutions to Eq. (8), the phase parameter equation.

As indicated above, we focus here on the simplest case of a spatially homogeneous medium with the real propagation constant $\beta_{0}$. Typically, this medium would be meant to represent free space. In this case Eqs. (6)-(8) reduce to

$$
\begin{aligned}
Q_{x}{ }^{2}+\beta_{0} \frac{\mathrm{d} Q_{x}}{\mathrm{~d} z} & =0, \\
Q_{x} S_{x}+\beta_{0} \frac{\mathrm{d} S_{x}}{\mathrm{~d} z} & =0, \\
\frac{\mathrm{d} P}{\mathrm{~d} z} & =-i \frac{Q_{x}}{2 \beta_{0}}-\frac{S_{x}{ }^{2}}{2 \beta_{0}} .
\end{aligned}
$$

We can also note from Eq. (10) that in this limit the complex displacement parameter is given in terms of the beam location by

$$
\begin{aligned}
S_{x}(z) & =-Q_{x}(z) d_{x a}(z)+\beta_{0} d_{x a}^{\prime}(0) \\
& =-Q_{x}(z) d_{x a}(0)+\left[\beta_{0}-Q_{x}(z) z\right] d_{x a}^{\prime}(0),
\end{aligned}
$$

where use has been made of the fact that in a uniform medium the rate of change of the amplitude displacement is constant, whereas the displacement itself varies linearly with $z$.

\section{Propagation in a Uniform Media}

In Section 2 a set of beam modes was derived that can describe the distribution of electromagnetic fields as they propagate in spatially homogeneous real dielectric media. In this process the partial differential wave equation was reduced to a set of ordinary differential equations. Our solutions are not complete until these secondary beam parameter equations have actually been solved. Thus it is now necessary to solve the coupled ordinary first-order differential equations given above as Eqs. (11)-(13).

One can readily show that the solution to Eq. (11) has the familiar form

$$
\frac{1}{q_{x}(z)}=\frac{1 / q_{x 1}}{1+z / q_{x 1}}
$$

where $1 / q_{x 1}$ is the initial $(z=0)$ value of $1 / q_{x}(z)$ and where we replace Eq. (9) with the standard low-gainper-wavelength form of the beam parameter:

$$
\frac{Q_{x}(z)}{\beta_{0}}=\frac{1}{q_{x}(z)}=\frac{1}{R_{x}(z)}-\frac{i \lambda}{n \pi w_{x}^{2}(z)} .
$$

In this expression $\lambda$ is the vacuum wavelength and $n$ is the index of refraction. With Eqs. (15) and (16), Eq. (12) can be rewritten in the form

$$
\frac{1}{S_{x}(z)} \frac{\mathrm{d} S_{x}(z)}{\mathrm{d} z}=-\frac{1 / q_{x 1}}{1+z / q_{x 1}} .
$$

One readily finds that the solution of this equation can be written as

$$
S_{x}(z)=\frac{S_{x 1}}{1+z / q_{x 1}}
$$

where $S_{x 1}$ is the initial value of $S_{x}(z)$. With Eqs. (15) and (18), the phase parameter equation given above as Eq. (13) takes the form

$$
\frac{\mathrm{d} P(z)}{\mathrm{d} z}=-\frac{i}{2} \frac{1 / q_{x 1}}{1+z / q_{x 1}}-\frac{S_{x 1}{ }^{2} / 2 \beta_{0}}{\left(1+z / q_{x 1}\right)^{2}} .
$$

This equation can also be integrated, and the result is

$$
P(z)=P_{1}-\frac{i}{2} \ln \left(1+\frac{z}{q_{x 1}}\right)-\frac{S_{x 1}^{2} q_{x 1}}{2 \beta_{0}} \frac{z / q_{x 1}}{1+z / q_{x 1}},
$$

where $P_{1}$ is the initial value of $P(z)$. When the various parameter formulas obtained here are introduced into Eq. (5), one has a complete description of the propagation of the fundamental off-axis Gaussian beam in real uniform media.

We can explore the significance and applications of these results more readily by focusing on specific cases. It follows from Eq. (16) that if the starting position of the beam is at the beam waist $\left(R_{x 1}=\infty\right)$ the beam parameter there must be purely imaginary. By convention we use the notation $1 / q_{x 1}=-i / z_{0}$, where $z_{0}$ is the Rayleigh length. With this substitution Eq. (15) becomes

$$
\frac{1}{q_{x}(z)}=\frac{-i / z_{0}}{1-i z / z_{0}} .
$$

When combined with Eq. (16), Eq. (21) yields the standard formulas for the propagation of the spot size and the phase-front curvature, whereas Eqs. (18) and (20) become

$$
\begin{aligned}
S_{x}(z) & =\frac{S_{x 1}}{1-i z / z_{0}}, \\
P(z) & =P_{1}-\frac{i}{2} \ln \left(1-\frac{i z}{z_{0}}\right)-\frac{S_{x 1}{ }^{2} z_{0}}{2 \beta_{0}} \frac{z / z_{0}}{1-i z / z_{0}} .
\end{aligned}
$$

As noted above, it is convenient to express the complex displacement parameter $S_{x}$ in terms of the actual displacement of the amplitude center of the beam $d_{x a}$ and the rate of change of this parameter 
with the propagation distance. Combining Eqs. (14), (16), and (21), one finds that the complex displacement parameter can be written as

$$
\begin{aligned}
S_{x}(z)= & -\frac{\beta_{0}}{z_{0}}\left[\frac{\left(z / z_{0}\right)-i}{1+\left(z / z_{0}\right)^{2}}\right]\left[d_{x a}(0)+\frac{z}{z_{0}} \frac{\mathrm{d} d_{x a}}{\mathrm{~d}\left(z / z_{0}\right)}\right] \\
& +\frac{\beta_{0}}{z_{0}} \frac{\mathrm{d} d_{x a}}{\mathrm{~d}\left(z / z_{0}\right)} \\
= & -\frac{2}{w_{0}^{2}}\left[\frac{\left(z / z_{0}\right)-i}{1+\left(z / z_{0}\right)^{2}}\right]\left[d_{x a}(0)+\frac{z}{z_{0}} \frac{\mathrm{d} d_{x a}}{\mathrm{~d}\left(z / z_{0}\right)}\right] \\
& +\frac{2}{w_{0}^{2}} \frac{\mathrm{d} d_{x a}}{\mathrm{~d}\left(z / z_{0}\right)},
\end{aligned}
$$

where the formula for the Rayleigh length in terms of the spot size at the beam waist $z_{0}=n \pi w_{0}^{2} / \lambda$ has been used to eliminate the propagation constant $\beta_{0}$. It is also convenient to normalize distances in the direction of propagation with respect to the Rayleigh length by introduction of the normalized distance $z^{\prime}$ $=z / z_{0}$. With this substitution Eq. (24) can be rewritten more compactly:

$S_{x}\left(z^{\prime}\right)=-\frac{2}{w_{0}^{2}}\left(\frac{z^{\prime}-i}{1+z^{\prime 2}}\right)\left(d_{x a}(0)+z^{\prime} \frac{\mathrm{d} d_{x a}}{\mathrm{~d} z^{\prime}}\right)+\frac{2}{w_{0}^{2}} \frac{\mathrm{d} d_{x a}}{\mathrm{~d} z^{\prime}}$.

Finally, it is also helpful to normalize the spot size and beam displacement to an arbitrary distance $d$ :

$$
S_{x}\left(z^{\prime}\right)=-2\left(\frac{z^{\prime}-i}{1+z^{\prime 2}}\right) \frac{p_{1}+z^{\prime} v_{1}}{w_{0}^{\prime 2} d}+2 \frac{v_{1}}{w_{0}^{\prime 2} d} .
$$

In this result the normalized spot size $w_{0}^{\prime}=w_{0} / d$, the initial position in the $x$ direction $p_{1}=d_{x a} / \mathrm{d}$, and the rate of change of this position with respect to normalized distance in the $z$ direction $v_{1}=\mathrm{d} p_{1} / \mathrm{d} z^{\prime}$ have been introduced. The initial value of the complex displacement parameter is needed for substitution in the phase parameter formulas, and from Eq. (26) this initial value is

$$
S_{x 1}=2 \frac{v_{1}+i p_{1}}{w_{0}^{\prime 2} d} .
$$

By use of Eq. (27) with simplifications similar to those used for the complex displacement parameter, the phase parameter result given in Eq. (23) takes the form

$$
\begin{aligned}
P\left(z^{\prime}\right) & =P_{1}-\frac{i}{2} \ln \left(1-i z^{\prime}\right)-\frac{z^{\prime}}{w_{0}^{\prime 2}}\left[\frac{\left(v_{1}+i p_{1}\right)^{2}}{1-i z^{\prime}}\right] \\
& =-\frac{i}{2}\left\{\ln \left(1+z^{\prime 2}\right)^{1 / 2}-i \tan ^{-1}\left(z^{\prime}\right)\right]-\frac{z^{\prime}}{w_{0}^{\prime 2}} \\
\times & \frac{\left[\left(v_{1}^{2}-p_{1}^{2}\right)-2 v_{1} p_{1} z^{\prime}\right]+i\left[\left(v_{1}^{2}-p_{1}^{2}\right) z^{\prime}+2 v_{1} p_{1}\right]}{1+z^{\prime 2}},
\end{aligned}
$$

where the initial value of the phase has been set arbitrarily to zero. Now the parameter functions given in Eqs. (21), (26), and (28) can be substituted into Eq. (5), and the field is found to be

$$
\begin{aligned}
A\left(x^{\prime}, z^{\prime}\right)= & A_{0} \exp \left\{\left[-\frac{1}{w_{0}^{\prime 2}\left(1+z^{\prime 2}\right)}-i \frac{z^{\prime}}{w_{0}^{\prime 2}\left(1+{z^{\prime}}^{2}\right)}\right] x^{\prime 2}\right. \\
& +\left[\frac{2\left(p_{1}+z^{\prime} v_{1}\right)}{w_{0}^{\prime 2}\left(1+z^{\prime 2}\right)}+i \frac{2 z^{\prime}\left(p_{1}+z^{\prime} v_{1}\right)}{w_{0}^{\prime 2}\left(1+z^{\prime 2}\right)}\right. \\
& \left.-i \frac{2 v_{1}}{w_{0}^{\prime 2}}\right] x^{\prime}+\left[-\ln \left(1+z^{\prime 2}\right)^{1 / 4}\right. \\
& -\frac{z^{\prime 2}\left(v_{1}^{2}-p_{1}^{2}\right)+2 z^{\prime} v_{1} p_{1}}{w_{0}^{\prime 2}\left(1+z^{\prime 2}\right)} \\
& +i \frac{z^{\prime}\left(v_{1}^{2}-p_{1}^{2}\right)-2{z^{\prime}}^{\prime 2} v_{1} p_{1}}{w_{0}^{\prime 2}\left(1+{z^{\prime}}^{2}\right)} \\
& \left.\left.+\frac{i}{2} \tan ^{-1}\left(z^{\prime}\right)\right]\right\},
\end{aligned}
$$

where the transverse coordinate has been normalized according to $x^{\prime}=\mathrm{x} / \mathrm{d}$.

It is convenient for many purposes to deal with the intensity of the beam rather than the amplitude. We define the intensity with the relation

$$
I\left(x^{\prime}, z^{\prime}\right)=A^{*}\left(x^{\prime}, z^{\prime}\right) A\left(x^{\prime}, z^{\prime}\right) .
$$

We also choose the amplitude coefficient $A_{0}$ of the off-axis Gaussian beam given in Eq. (29) so that $I\left(x^{\prime}\right.$, $z^{\prime}$ ) will be normalized according to the integral

$$
\int_{-\infty}^{\infty} I\left(x^{\prime}, z^{\prime}\right) \mathrm{d} x^{\prime}=1
$$

After some arithmetic one finds that the normalized field can be written as

$$
\begin{aligned}
A\left(x^{\prime}, z^{\prime}\right)= & {\left[\frac{(2 / \pi)^{1 / 2}}{w_{0}^{\prime}\left(1+{z^{\prime}}^{\prime 2}\right)^{1 / 2}}\right]^{1 / 2} \exp \left[-\frac{1}{w_{0}^{\prime 2}\left(1+z^{\prime 2}\right)} x^{\prime 2}\right.} \\
& \left.+\frac{2\left(p_{1}+z^{\prime} v_{1}\right)}{w_{0}^{\prime 2}\left(1+z^{\prime 2}\right)} x^{\prime}-\frac{p_{1}^{2}+2 z^{\prime} v_{1} p_{1}+z^{\prime 2} v_{1}^{2}}{w_{0}^{\prime 2}\left(1+z^{\prime 2}\right)}\right] \\
& \times \exp \left(-i\left\{\frac{z^{\prime}}{w_{0}^{\prime 2}\left(1+z^{\prime 2}\right)} x^{\prime 2}\right.\right. \\
& +\left[\frac{2 v_{1}}{w_{0}^{\prime 2}}-\frac{2 z^{\prime}\left(p_{1}+z^{\prime} v_{1}\right)}{w_{0}^{\prime 2}\left(1+z^{\prime 2}\right)}\right] x^{\prime} \\
& \left.\left.-\frac{z^{\prime}\left(v_{1}^{2}-p_{1}^{2}\right)-2 z^{\prime 2} v_{1} p_{1}}{w_{0}^{\prime 2}\left(1+z^{\prime 2}\right)}-\frac{1}{2} \tan ^{-1}\left(z^{\prime}\right)\right\}\right) \\
= & \left.\frac{(2 / \pi)^{1 / 2}}{w_{0}^{\prime}\left(1+z^{\prime 2}\right)^{1 / 2}}\right]^{1 / 2} \\
& \times \exp \left\{-\left[\frac{x^{\prime}-\left(p_{1}+z^{\prime} v_{1}\right)}{w_{0}^{\prime}\left(1+z^{\prime 2}\right)^{1 / 2}}\right]\right\} \\
& \times \exp \left(-i\left\{z^{\prime}\left[\frac{x^{\prime}-\left(p_{1}+z^{\prime} v_{1}\right)}{w_{0}^{\prime}\left(1+z^{\prime 2}\right)^{1 / 2}}\right]^{2}\right.\right. \\
& \left.\left.+\frac{2 v_{1} x^{\prime}-z^{\prime} v_{1}^{2}}{w_{0}^{\prime 2}}-\frac{1}{2} \tan ^{-1}\left(z^{\prime}\right)\right\}\right)
\end{aligned}
$$


Equation (32) with its preceding definitions is a complete solution for an off-axis Gaussian beam that propagates in a real spatially homogeneous medium.

\section{Grazing Reflection}

We now have an analytical description of a Gaussian beam that propagates displaced and at a small angle with respect to an arbitrary $z$ axis. This analysis has not yet included the possibility of a reflecting surface in the beam path, but it is possible to start from this solution and determine the fields in the vicinity of such a surface. The basic idea is to use a superposition of solutions of the type given above. Because the wave equation as employed here is linear, any superposition of solutions is also a solution. Although the method to be described is applicable for arbitrary angles of incidence, our emphasis is on grazing reflections. The math is the simplest in this case, and there is also the greatest likelihood of practical applications. As noted above for grazingincidence, reflection is highest and the interference structure of the fields achieves its greatest spatial extent in comparison with the dimensions of the beams. The paraxial beams derived above are directly applicable in this case.

It is assumed here that the reflecting surface is a flat high reflector as might be achieved with a metal surface for grazing angles of incidence. The electromagnetic boundary conditions for such a surface are that the tangential component of the electric field must go to zero at the boundary. Thus for fields polarized parallel to the surface the amplitude of the fields must go to zero at the boundary. We refer to this as the parallel polarization (electric-field vector normal to the plane of incidence). For the perpendicular polarization (electric-field vector in the plane of incidence), the field might have a local maximum at the boundary. It follows from these considerations that a representation for a reflecting parallelpolarized Gaussian beam field requires that we find some superposition of Gaussian beams such that the fields all cancel at the location of the boundary. Such a superposition would satisfy the MaxwellHeaviside equations and would also by design satisfy the only boundary condition of the problem. For the case considered here the necessary superposition has only two components.

To be specific, it is now assumed that the reflecting surface is exactly parallel to the $z$ axis, whereas the beam itself might be propagating at a small angle with respect to this axis, as shown in Fig. 1. Initially, we also focus on parallel-polarized beams. For this case, one finds that an amplitude superposition that satisfies the boundary conditions is

$$
A_{\|}\left(x^{\prime}, z^{\prime}\right)=A\left(x^{\prime}, z^{\prime}\right)-A\left(1-x^{\prime}, z^{\prime}\right) .
$$

It is clear from this formula that at an $x^{\prime}$ value of $1 / 2$ the two components of the superposition cancel. Physically, this formula represents two Gaussian beams that are images of each other and for which the respective $z$ axes are separated by the distance $\mathrm{d}$.
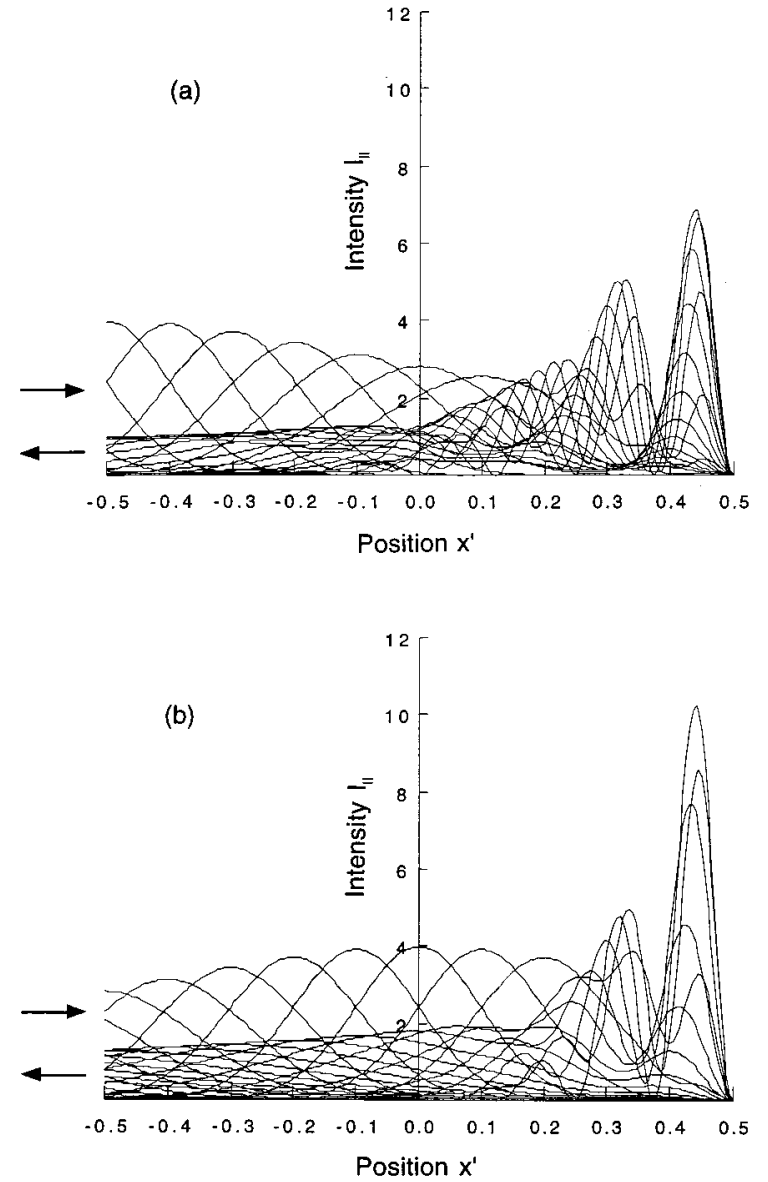

Fig. 2. Series of transverse intensity profiles of a normalized Gaussian beam undergoing grazing reflection from a flat surface located at the position $x^{\prime}=0.5$ on the right-hand side of the figure. The arrows to the left-hand side of the figure distinguish between the propagation directions of the more localized incident beam profiles (right arrow) and the less localized reflected beam profiles (left arrow). The beam is polarized parallel to the surface, the waist spot size is $w_{0}^{\prime}=0.2$, the velocity of the input beam toward the surface is $v_{1}=0.5$, and the propagation distance between successive profiles is $z^{\prime}=0.2$ (five plots per Rayleigh length). In (a) the beam waist (maximum beam-axis intensity) occurs just as the beam axis crosses the location $x^{\prime}=-0.5$. Because of increasing diffraction, the reflected beam in this example has a lower intensity and greater width than the incident beam. In (b) the waist occurs at $x^{\prime}=0.0$.

This distance was also chosen above as the normalization distance for the transverse beam and coordinate variables. In the grazing-reflection problem, the real space of interest occurs for $x^{\prime}$ values less than $1 / 2$.

It is more convenient to focus now on the intensities associated with the fields rather than on their amplitudes. For the parallel polarization the intensity from Eq. (30) is, of course, defined by

$$
I_{\|}\left(x^{\prime}, z^{\prime}\right)=A_{\|}^{*}\left(x^{\prime}, z^{\prime}\right) A_{\|}\left(x^{\prime}, z^{\prime}\right),
$$

and Eqs. (32)-(34) are the basis for Figs. 2 and 3. Figure 2 is a plot of a series of transverse intensity profiles for a normalized Gaussian beam that is un- 
dergoing grazing reflection from a flat surface located at the position $x^{\prime}=0.5$. The beam in this case is polarized parallel to the surface, the waist spot size is $w_{0}^{\prime}=0.2$, the velocity of the input beam toward the surface is $v_{1}=0.5$, and the propagation distance between successive profiles is $z^{\prime}=0.2$ (five plots per Rayleigh length). This display format shows in a compact way the changes in beam intensity and width that occur with propagation, and the arrows distinguish the right-traveling incident profiles from the left-traveling reflected profiles. As required by the boundary condition, the intensity goes to zero at the reflecting surface. In part (a) the position parameter $p_{1}$ has the value -0.5 , so that the beam waist (maximum beam-axis intensity) occurs just as the beam axis crosses the location $x^{\prime}=-0.5$. Because of increased diffraction, the reflected beam in this example has a lower intensity and greater width than the incident beam. In part (b) the waist occurs at $x^{\prime}$ $=0.0$. A striking feature of the results shown in Fig. 2 is the substantial increase in intensity as the beam interacts with the surface. This increase suggests that there could be an enhancement of any nonlinear interactions between beams if the interaction region were in the vicinity of a reflecting surface. The thickness of the interaction region and the effective phase velocity can also be controlled by variation of the beam orientations. Such adjustments might prove useful in nonlinear optical applications.

Figure 3 shows a plot of the transverse intensity profiles of a reflecting Gaussian beam whose waist occurs at the reflecting surface. The beam is again polarized parallel to the surface, and the waist spot size is $w_{0}^{\prime}=0.2$, but in this case the propagation distance between successive profiles is $z^{\prime}=0.1$. In part (a) the incident beam moves toward the surface at a velocity of $v_{1}=0.5$, and in part (b) the velocity of the beam is $v_{1}=1.0$. Because of the symmetry that occurs in this case, the reflected beam has the same intensity profile as the incident beam. It can be seen from these examples that changing the transverse velocity of the incident beam (or rather its propagation direction with respect to the $z$ axis) changes the interference structure near the surface. Higher velocities (more nearly normal incidence) leads to thinner interference structures.

Similar results are obtained for the intensity of a beam that is polarized perpendicular to the reflecting surface. Instead of Eq. (33) the reflection in this case can be represented by the alternative superposition

$$
A_{\perp}\left(x^{\prime}, z^{\prime}\right)=A\left(x^{\prime}, z^{\prime}\right)+A\left(1-x^{\prime}, z^{\prime}\right) .
$$

For this polarization the intensity is defined by

$$
I_{\perp}\left(x^{\prime}, z^{\prime}\right)=A_{\perp}^{*}\left(x^{\prime}, z^{\prime}\right) A_{\perp}\left(x^{\prime}, z^{\prime}\right),
$$

and Eqs. (32), (35), and (36) are the basis for Figs. 4 and 5. Figure 4 shows a plot of a reflecting beam for which the waist spot size is $w_{0}^{\prime}=0.2$, the velocity of the input beam toward the surface is $v_{1}=0.5$, and the propagation distance between successive profiles is $z^{\prime}$
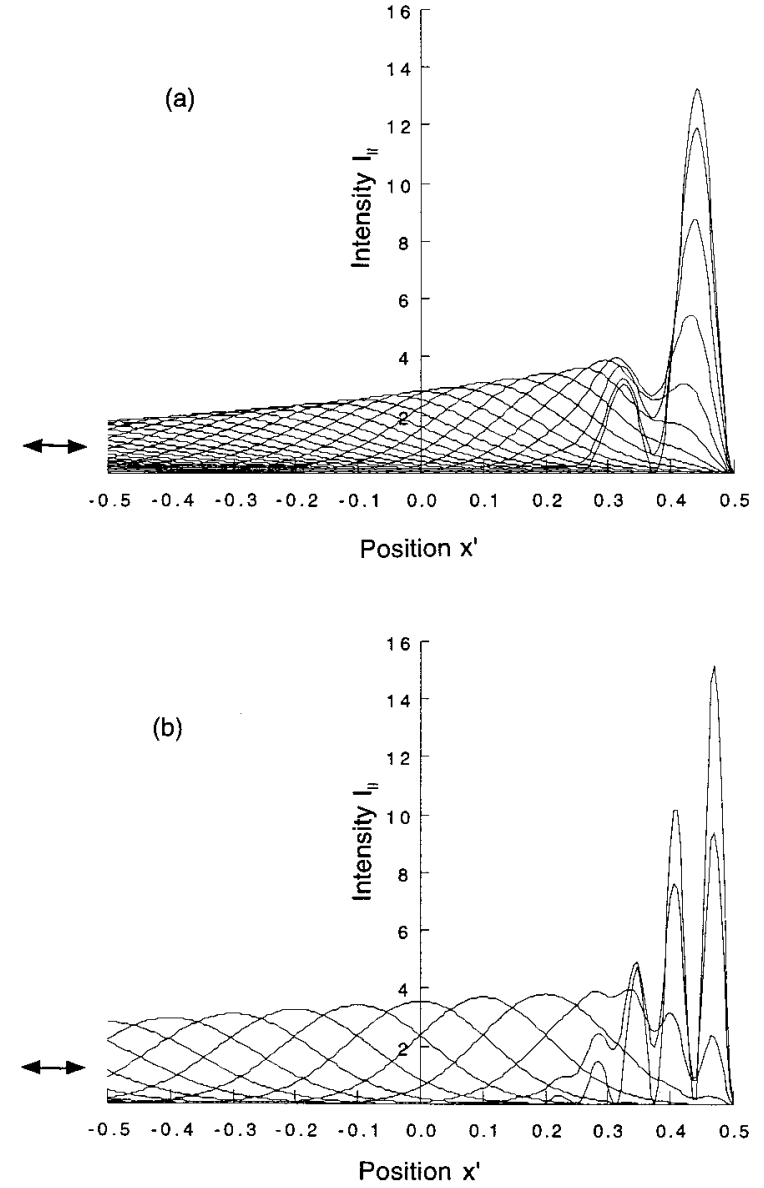

Fig. 3. Transverse intensity profiles of a reflecting Gaussian beam whose waist occurs at the reflecting surface. The twoheaded arrows to the left-hand sides of the figures indicate that in these cases the incident and reflected beam profiles are identical. The beam is polarized parallel to the surface, the waist spot size is $w_{0}^{\prime}=0.2$, and the propagation distance between successive profiles is $z^{\prime}=0.1$. In (a) the incident beam is moving toward the surface at a velocity of $v_{1}=0.5$, and in (b) the velocity of the beam is $v_{1}=$ 1.0 .

$=0.2$. In part (a) the beam waist occurs as the beam axis crosses the location $x^{\prime}=-0.5$, and in part (b) the waist is at $x^{\prime}=0.0$. In contrast to Fig. 2 , the beams in Fig. 4 have a maximum of their intensity at the reflecting surface. For the beams in Fig. 5 the waist occurs at the reflecting surface. The waist spot size is $w_{0}^{\prime}=0.2$, and the propagation distance between successive profiles is $z^{\prime}=0.1$. In part (a) the incident beam moves toward the surface at a velocity of $v_{1}=0.5$, and in part (b) the velocity of the beam is $v_{1}$ $=1.0$.

\section{Discussion}

The high reflectivities that occur when beams are incident at grazing angles have been the basis for many practical applications, particularly in wavelength regions of the spectrum where other highly reflecting surfaces are not readily available. Early applications occurred in spectroscopy, and curved single-sided waveguides have also been used for 

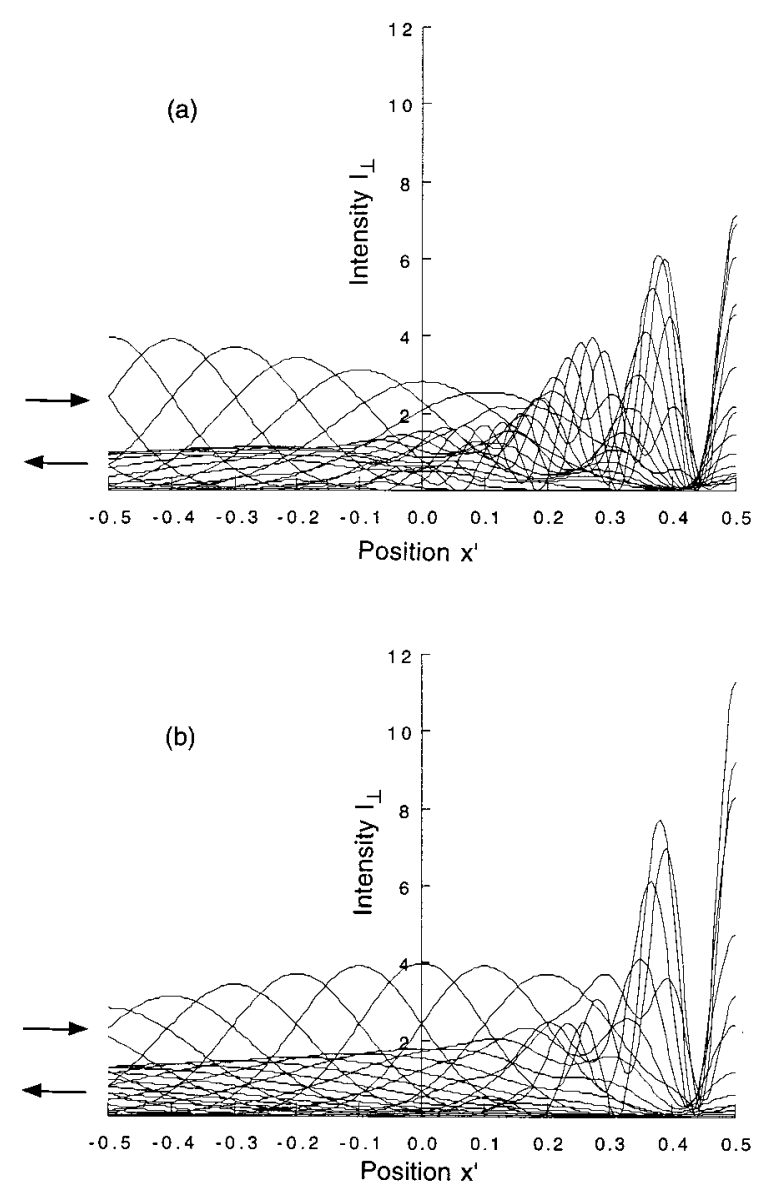

Fig. 4. Transverse intensity profiles of a reflecting Gaussian beam. The beam is polarized perpendicular to the surface, the waist spot size is $w_{0}^{\prime}=0.2$, the velocity of the input beam toward the surface is $v_{1}=0.5$, and the propagation distance between successive profiles is $z^{\prime}=0.2$. In (a) the beam waist occurs as the beam axis crosses the location $x^{\prime}=-0.5$, and in (b) the waist is at $x^{\prime}=0.0$.

beam transmission. Recent examples have involved Gaussian beams as the intracavity modes in lasers that incorporate grazing-incidence mirrors or as the pump beams in laser systems that use grazingincidence focusing optics. In this study analytic solutions of the paraxial wave equation for Gaussian beams that reflect at grazing angles from a flat surface have been obtained.

The results developed here should be useful if one wishes to understand or apply the detailed amplitude or intensity distribution of a Gaussian beam in the vicinity of a reflecting surface. Of particular interest is the much higher intensity that can occur owing to the interference of the incident and the reflected beams. A related intensity layer was used previously to provide efficient coupling to the thin gain region in certain electrode-guided gas lasers. This layer has led to enhanced resolution in scanning optical microscopy, and it might also be useful in nonlinear optics. Depending on the design and orientation of the reflecting surface, one might have control over both the intensity distribution and the
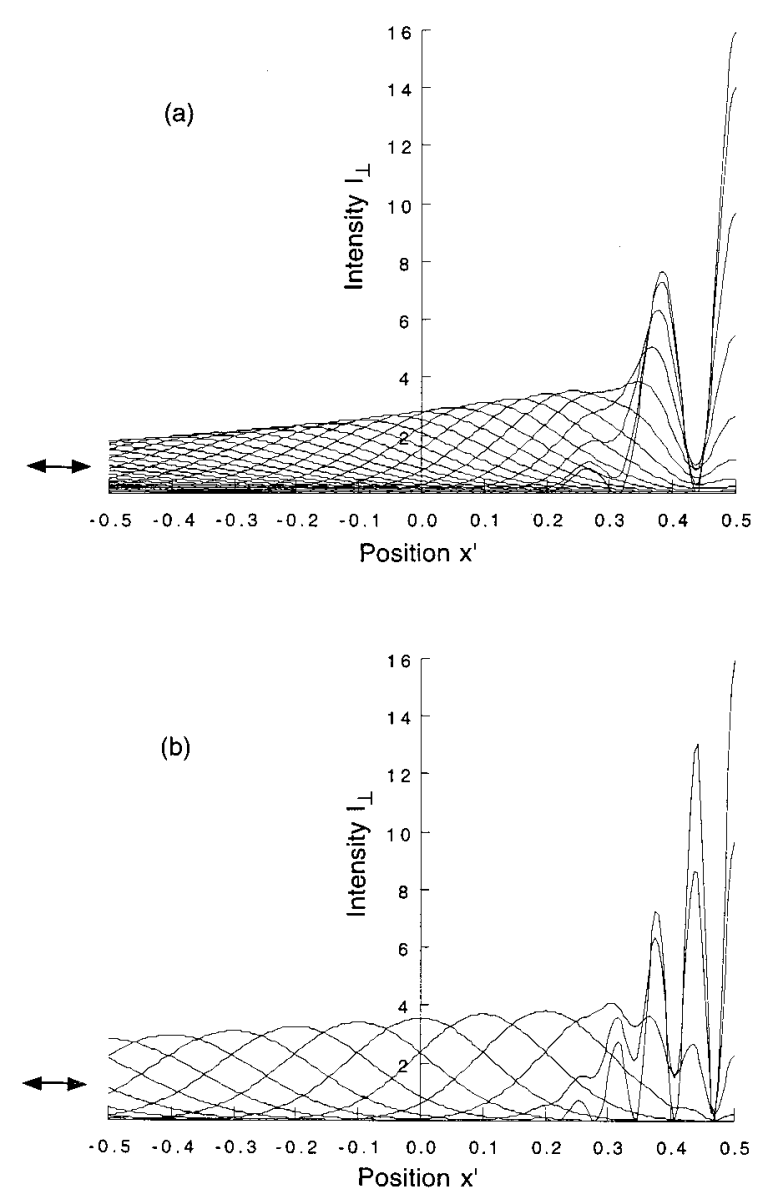

Fig. 5. Transverse intensity profiles of a reflecting Gaussian beam whose waist occurs at the reflecting surface. The beam is polarized perpendicular to the surface, the waist spot size is $w_{0}^{\prime}=$ 0.2 , and the propagation distance between successive profiles is $z^{\prime}$ $=0.1$. In (a) the incident beam is moving toward the surface at a velocity of $v_{1}=0.5$, and in (b) the velocity of the beam is $v_{1}=1.0$.

phase velocity of the propagating beams near the surface.

This research was supported in part by the National Science Foundation under grant PHY9415583. The author also expresses his appreciation to the members of the Rochester Theory Center for Optical Science and Engineering and the Institute of Optics at the University of Rochester for valuable discussions and hospitality during his sabbatical visit.

\section{References}

1. G. D. Boyd and J. P. Gordon, "Confocal multimode resonator for millimeter through optical wavelength masers," Bell Sys. Tech. J. 40, 489-508 (1961).

2. H. Kogelnik, "Imaging of optical modes-resonators with internal lenses," Bell Sys. Tech. J. 44, 455-494 (1965).

3. H. Kogelnik, "On the propagation of Gaussian beams of light through lenslike media including those with a loss or gain variation," Appl. Opt. 4, 1562-1569 (1965).

4. L. W. Casperson and S. D. Lunnam, "Gaussian modes in high loss laser resonators,” Appl. Opt. 14, 1193-1199 (1975), and references therein. 
5. L. W. Casperson, "Beam modes in complex lenslike media and resonators,” J. Opt. Soc. Am. 66, 1373-1379 (1976).

6. A. A. Tovar and L. W. Casperson, "Generalized beam matrices: Gaussian beam propagation in misaligned complex optical systems," J. Opt. Soc. Am. 12, 1522-1533 (1995).

7. W. S. Bacsa and A. Kulik, "Interference scanning optical probe microscopy," Appl. Phys. Lett. 70, 3507-3509 (1997).

8. See, for example, J. A. R. Samson, Techniques of Vacuum Ultraviolet Spectroscopy (Wiley, New York, 1967), Section 2.8 and references therein.

9. See, for example, A. N. Zaidel and E. Y. Shreider, Vacuum Ultraviolet Spectroscopy (Ann Arbor-Humphrey Science Publishers, Inc., Ann Arbor, Mich., 1970), pp. 119-126.

10. H. Krammer, "Light waves guided by a single curved metallic strip," Appl. Opt. 17, 316-319 (1978).

11. L. W. Casperson and T. S. Garfield, "Guided beams in concave metallic waveguides," IEEE J. Quantum Electron. QE-15, 491-496 (1979), and references therein.

12. M. E. Marhic, L. I. Kwan, and M. Epstein, "Whispering-gallery $\mathrm{CO}_{2}$ laser," IEEE J. Quantum Electron. QE-15, 487-490 (1979).

13. J. G. Grossman, L. W. Casperson, and O. M. Stafsudd, "Radiofrequency-excited carbon dioxide metal waveguide laser," Appl. Opt. 22, 1298-1305 (1983).

14. F. S. Al-Mashaabi and L. W. Casperson, "Direct currentexcited cw $\mathrm{CO}_{2}$ metal waveguide laser," Appl. Opt. 28, 18971903 (1989).

15. J. M. Eggleston, "Angularly stable ring resonators for high power FELs," in Proceedings of the International Conference on Lasers 1983, R. Powell, ed. (STS, McLean, Va., 1985), p. 305.

16. E. Sklar, "The tilt sensitivity of a grazing incidence confocal unstable resonator with applications to free-electron lasers," IEEE J. Quantum Electron. QE-23, 229-233 (1987).
17. K. C. Sun, "Grazing incidence ring resonator for visible wavelength free electron laser," in Optical Resonators, D. A. Holmes, ed., Proc. SPIE 1224, 409-422 (1990).

18. D. H. Dowell, M. L. Laucks, A. R. Lowrey, J. L. Adamski, D. J. Pistoresi, D. R. Shoffstall, M. P. Bentz, R. H. Burns, J. Guha, K. C. Sun, W. Tomita, A. H. Lumpkin, S. C. Bender, D. Byrd, and R. L. Tokar, "Tests of a grazing-incidence ring resonator free-electron laser," IEEE J. Quantum Electron. 27, 26132625 (1991).

19. D. H. Dowell, M. L. Laucks, A. R. Lowrey, J. L. Adamski, D. J. Pistoresi, D. R. Shoffstall, A. H. Lumpkin, S. Bender, D. Byrd, R. L. Tokar, K. Sun, M. Bentz, R. Burns, J. Guha, and W. Tomita, "Final results of the Boeing and Los Alamos grazing incidence ring-resonator free electron laser experiment," Nucl. Instrum. Methods A 318, 74-80 (1992).

20. D. R. Gabardi and D. L. Shealy, "Optical analysis of grazing incidence ring resonators for free-electron lasers," Opt. Eng. 29, 641-648 (1990).

21. M. C. Wang, Y. Yuan, and Z. Wang, "The grazing incidence ring resonator for a free-electron laser," Nucl. Instrum. Methods A 318, 874-876 (1992).

22. J. P. Braud, "Laser cavities and polarization optics for soft X-rays and the extreme ultraviolet," Appl. Phys. B 50, 205-212 (1990).

23. J. F. Young, J. J. Macklin, and S. E. Harris, “Grazing-incidence ellipsoidal reflector for longitudinally pumping shortwavelength lasers," Opt. Lett. 12, 90-92 (1987).

24. L. W. Casperson, "Gaussian light beams in inhomogeneous media," Appl. Opt. 12, 2434-2441 (1973).

25. A. A. Tovar and L. W. Casperson, "Generalized beam matrices: Gaussian beam propagation in misaligned complex optical systems," J. Opt. Soc. Am. A 12, 1522-1533 (1995), Eq. (24). 